\title{
Sharing Emotions and Space - Empathy as a Basis for Cooperative Spatial Interaction
}

\author{
Hana Boukricha, Nhung Nguyen, and Ipke Wachsmuth` \\ A.I. Group, Faculty of Technology \\ Bielefeld University \\ 33594 Bielefeld, Germany \\ \{hboukric,nnguyen, ipke\}@techfak.uni-bielefeld.de
}

\begin{abstract}
Empathy is believed to play a major role as a basis for humans' cooperative behavior. Recent research shows that humans empathize with each other to different degrees depending on several modulation factors including, among others, their social relationships, their mood, and the situational context. In human spatial interaction, partners share and sustain a space that is equally and exclusively reachable to them, the so-called interaction space. In a cooperative interaction scenario of relocating objects in interaction space, we introduce an approach for triggering and modulating a virtual humans cooperative spatial behavior by its degree of empathy with its interaction partner. That is, spatial distances like object distances as well as distances of arm and body movements while relocating objects in interaction space are modulated by the virtual human's degree of empathy. In this scenario, the virtual human's empathic emotion is generated as a hypothesis about the partner's emotional state as related to the physical effort needed to perform a goal directed spatial behavior.
\end{abstract}

\section{Introduction and Motivation}

In human social interaction, empathy plays a major role as a motivational basis of cooperative behavior and as contributing to moral acts like helping, caring, and justice [11]. Recent neuropsychological findings [6] substantiate that empathic brain responses are prone to modulation and thus humans empathize with each other to different degrees. The modulation depends on several factors including, among others, humans' social relationships, their mood, and the situational context. In human spatial cooperation the interactants share and sustain a space that is equally and exclusively reachable to them [13]. In such interaction the partners' reach-spaces, the so called peripersonal spaces, may overlap and establish a shared reach-space defined as their interaction space [19]. Previous works have shown that virtual humans are a suitable testbed to study empathic behavior, e.g., [23] and spatial behavior, e.g., [22]. In this paper, we introduce an approach to trigger and modulate a virtual human's cooperative spatial behavior by its degree of empathy with its interaction partner.

In a spatial interaction scenario of a tower building task, potential field functions are used to control the spatial actions of the virtual human Max [15] in peripersonal

\footnotetext{
* Authors are listed in alphabetical order.
} 
and interaction space. Depending on the size and layout of the interaction space, Max can relocate objects to any free location toward or away from locations reachable for its partner [19]. Since in this scenario, Max's cooperative spatial behavior is a helping action that consists of relocating objects toward positions reachable for the partner, the question arises which position within interaction space is chosen to help the partner. In order to deal with this question, Max's helping action is triggered and modulated by its degree of empathy with its partner. That is, spatial distances like object distances as well as distances of arm and body movements while relocating objects toward positions reachable for the partner are modulated by Max's degree of empathy with its partner. Max's empathic behavior consists of three processing steps [5]: First, the Empathy Mechanism by which an empathic emotion is generated as a hypothesis about the partner's emotional state as related to the physical effort needed to perform a goal directed spatial behavior. Second, the Empathy Modulation by which the empathic emotion is modulated through modulation factors like Max's mood, relationship to the partner, and the situational context. Third, the Expression of Empathy by which Max's modulated facial expression and helping action are triggered.

The paper is structured as follows: In Section 2, related work on virtual humans exhibiting empathic and spatial behaviors are outlined. In Section 3, Max's spatial behavior is described. In Section 4, we present the cooperative spatial interaction task. In Section 5, Max's empathic behavior is described. Subsequently in Section 6, we introduce an approach for modulating Max's cooperative spatial behavior. Finally in Section 7 , we give a summary of the main aspects underlying our approach and an outlook on future work.

\section{Related Work}

There are various attempts to endow virtual humans with the ability to empathize. McQuiggan et al. [18] propose an inductive framework for modeling parallel and reactive empathy in virtual agents. Their framework is called CARE (Companion Assisted Reactive Empathizer) and is based on learning empirically grounded models of empathy from observing human-agent social interactions. In a virtual training environment, users are able to evaluate the virtual character's empathic reaction allowing it to learn models of empathy from "good" examples. Based on an empirical and theoretical approach Ochs et al. [20] propose a computational model of empathic emotions. The empirical part is based on analyzing human-machine dialogs in order to identify the characteristics of emotional dialog situations. The theoretical part is based on cognitive psychological theories and consists of determining the type and intensity of the empathic emotion. In [24], the empathy model is implemented into an affective agent architecture and the intensity of the empathic emotion is determined by the following modulation factors: similarity, affective link, mood, and personality. Boukricha and Wachsmuth [5], presented a computational model of empathy. Within this model, the type and intensity of the empathic emotion are modulated by factors like the virtual human's mood and relationship to its interaction partner. In a scenario of interactive affective narratives, Aylett and Louchart [1] use a double appraisal mechanism in order to determine the emotional 
impact of an action on the other characters. Double appraisal means using a characters' own appraisal mechanisms for reasoning about the emotions of others.

Cooperative behavior of robots and virtual humans in task execution with humans was presented in previous works. In work by Kopp et al. [15] a virtual human performs a construction task with a human partner in a face-to-face virtual reality scenario. The virtual human cooperates with his partner by describing assembly plans and by offering verbal and gestural assistance on request. In another scenario, where a robot is to support human partners in building wooden construction toys, Foster et al. [7] introduced a dialogue system which provides the robot with abilities similar to Max's. In work of Gray et al. [9] a robot performs a button-push task in cooperation with a human partner. The robot uses its own motor-action repertoire to recognize the partner's actions in order to infer the partner's goals and offer help, such as completing a failed action. With a focus on the spatial aspect of actions within cooperative task execution, Nguyen and Wachsmuth [19] presented a model for structuring and controlling a virtual human's spatial behavior and attention in task execution at close distances. Apart of such execution tasks, spatial behavior models have been presented in locomotion scenarios for robots and virtual humans. Pedica and Vilhjálmsson [22] for example controlled virtual humans' spatial group formations in virtual reality environments like games. Sisbot et al. [25] presented their work on robot locomotion planning considering factors like humans' comfort, preferences, and safety.

While significant advances have been made in modeling the empathic, spatial, and cooperative behaviors of virtual humans, triggering and modulating a virtual human's cooperative spatial behavior by its degree of empathy was not addressed so far. In the present work, the virtual human's degree of empathy is affected by factors like its mood, its relationship to the partner, and the situational context. The virtual human's Empathy Mechanism is based on using its own appraisal mechanisms for generating an empathic emotion. This process is similar to those mentioned above in [9] and [1].

\section{Spatial Behavior}

In this section we outline how the space surrounding an individual virtual human and the space in interpersonal interaction is modeled. The surrounding spaces are projected on an assumed 2-D plane on a table in front of the virtual human, in this case Max. Hence, the vertical extent of each space is projected on a lower radial $180^{\circ} 2$-D plane. Furthermore, the table size needs to assure sufficient space for object manipulation and for both partners to lean forward and carry out arm movements without too much obstruction.

\subsection{Peripersonal and Interaction Space}

The work on peripersonal space is motivated by research from biology and cognitive neuroscience. The reach- and lean-forward distances are calculated from Max's body structure [19]. The peripersonal space is divided into subspaces differing in spatial range, extent and frames of reference. In this paper we focus on the touch space and the lean-forward space (see Figure 1, left). The touch space's boundary is limited to the 
lengths of the arm limbs. The lean-forward space's boundary is limited to the maximal reaching realm of the upper torso when bending forward. Another agent (human or virtual) entering Max's proximity is assumed to be surrounded by a peripersonal space in the same way as Max. The intersection of their overlapping peripersonal spaces is what was defined in previous work [19] as interaction space (see Figure 1, right). This space is equally and exclusively reachable to the interactants, and is the space in which they cooperate.

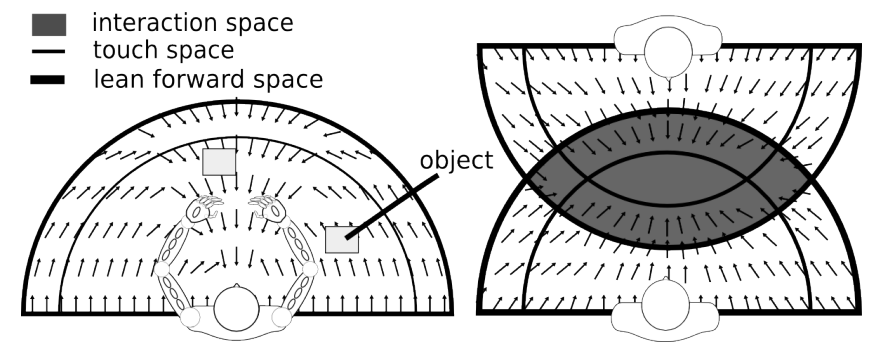

Fig. 1. Left: Subspaces surrounding a virtual human. Lean-forward space as an extension of touch space. Arrows illustrate force vectors in a repulsive artificial potential field, here pointing to the front of the body. Right: Interaction space spanned by two partners.

\subsection{Potential Fields}

In order to trigger appropriate motor actions with regard to objects at each location in peripersonal and interaction space, we use artificial potential fields [14], a common method for obstacle avoidance and path planning for artificial agents. The peripersonal space is described as a repulsive field. A vector between the center of peripersonal space (i.e. an assumed center of Max's body) and any position $P$ in space is denoted by position vector $\mathbf{p}$. The touch space's field $F_{\text {touch }}$ is defined by Equation ?? with tangential directions covering a semicircle in front of Max's torso, defined by Equation 1 and illustrated in Figure 1, left. We calculate the force vector $\mathbf{v}_{\text {touch }}(\mathbf{p})$, that is currently affecting $\mathbf{p}$, using Equation 1. The parameter $\xi_{\text {peri }}$ denotes a positive scalar which influences the length of the resulting force vector. The force vectors $\mathbf{v}_{\text {touch }}(\mathbf{p})$ point to the frontal, sagittal midline of Max's body, described by vector $\mathbf{r}_{\text {perimid }}$. This midline defines a default direction in front of Max's body. The field covers all p's within an angle of $90^{\circ}$ to both sides of this midline. The regions beyond the radius $r_{\text {touch }}$ of touch space are not affected by the potential field, and thus result in a zero force vector. The lean-forward space is located between $r_{\text {touch }}$ and a larger radius $r_{\text {lean }}$. It is modeled as an extension of the touch space, thus the potential field $F_{\text {touch }}$ can be extended up to $r_{l e a n}$. Regions beyond $r_{\text {lean }}$ belong to extrapersonal space. The interaction space is modeled as an attractive potential field. Since interaction space affects the peripersonal spaces of both partners, its radius includes both involved peripersonal potential fields. All forces currently affecting any position $P$ in space have to be summed up to obtain the resulting force. Each time Max perceives an object, the current resulting force vector impacting 
on the object has to be calculated. Objects outside the interaction space are affected by force vectors within the peripersonal space, describing a path which leads in the direction of the interaction space. With decreasing distance to the center, the strength of the potential field disappears, ending the path.

$$
\begin{gathered}
\mathbf{F}_{\text {touch }}(\mathbf{p})= \begin{cases}\xi_{\text {peri }}\left(\frac{1}{\|\mathbf{p}\|}-\frac{1}{r_{\text {touch }}}\right) \frac{\mathbf{p}}{\|\mathbf{p}\|^{3}} & \|\mathbf{p}\| \leq r_{\text {touch }}, \\
0 & \text { else }\end{cases} \\
\mathbf{v}_{\text {touch }}(\mathbf{p})= \begin{cases}-\left(\frac{\pi}{2}\right) * \mathbf{F}_{\text {touch }}(\mathbf{p}) & \forall \mathbf{p} \mid \measuredangle\left(\mathbf{r}_{\text {perimid }}, \mathbf{p}\right) \leq-\left(\frac{\pi}{2}\right), \\
\left(\frac{\pi}{2}\right) * \mathbf{F}_{\text {peri }}(\mathbf{p}) & \forall \mathbf{p} \mid \measuredangle\left(\mathbf{r}_{\text {perimid }}, \mathbf{p}\right) \leq\left(\frac{\pi}{2}\right), \\
0 & \text { else }\end{cases}
\end{gathered}
$$

\subsection{Potential Field Parameters for Modulating Spatial Actions and Distances}

Potential fields are a suitable method to associate each point in peripersonal space to a specific behavior, in this case motor actions. By superposing several potential fields, behaviors can be combined, allowing for more sophisticated actions like reaching with collision avoidance. Another way to influence Max's spatial behavior is by changing the parameters of the potential field of Max's touch space (see Equation ??). The following parameters are influenced by Max's degree of empathy (see Section 6).

Field strength The field strength parameter $\xi_{\text {peri }}$ in equation ?? determining the lengths of the resulting force vectors controls the velocity of a chosen motor action.

Field radius The field radius parameter $r_{\text {touch }}$ in equation ?? determines the end point of the chosen motor action. The maximum value for $r_{\text {touch }}$ is determined by $r_{\text {lean }}$, which results in motor actions within the lean-forward space.

\section{Cooperative Building Task}

In a virtual reality CAVE-like environment Max and his interaction partner are standing face-to-face at a table in order to solve a cooperative building task with virtual toy blocks. According to Section 3 the partner's overlapping peripersonal spaces form an interaction space.

The goal of the spatial interaction task is to cooperatively build a tower by alternately putting a toy block one upon the other. All tower blocks are labeled with numbers and differ in their size. The numbers ascend with larger size, i.e, the largest block is labeled with the highest number. At the beginning of the game the largest block is placed by default in the center of the partners' interaction space, where they have to place the remaining blocks. The remaining blocks are randomly placed at free locations within the partners' peripersonal spaces. Each partner may get a different number of blocks with respect to a predefined minimum. There are two rules to build a tower: First, the tower blocks can be ordered by their number-labels, e.g., block number two is put on block number three. When all existing blocks are ordered by their numbers, this leads to the highest ideal tower. Second, the tower blocks can be ordered by their sizes without matching the direct number order, e.g., block number two is put on block number four, 
omitting block number three. This leads to a smaller tower. Each partner should place the most appropriate block located in his peripersonal space on the tower. The most appropriate block is defined as the one that best fits the above introduced rules. The tower building task ends when the smallest block is placed on the top of the tower.

The Virtual Human Max has a cognitive architecture composed of a Belief-DesireIntention (BDI) module [16] and of an emotion simulation module [3]. Based on domainspecific as well as domain-independent appraisal mechanisms, emotional valences are derived in the deliberative component of the BDI module [2] and drive Max's emotion dynamics over time, e.g., achieving a desired goal is rewarded with positive values of emotional valence. The emotion simulation module consists of two components: First, the dynamics/mood component for the calculation of the course of emotions and moods over time and their mutual interaction. Second, the Pleasure-Arousal-Dominance (PAD) space in which primary and secondary emotions are located and their intensity values are calculated. At each point in time, the emotion module outputs values of pleasure, arousal, and one of two possible values of dominance (dominant vs. submissive) as well as intensity values of primary and secondary emotions.

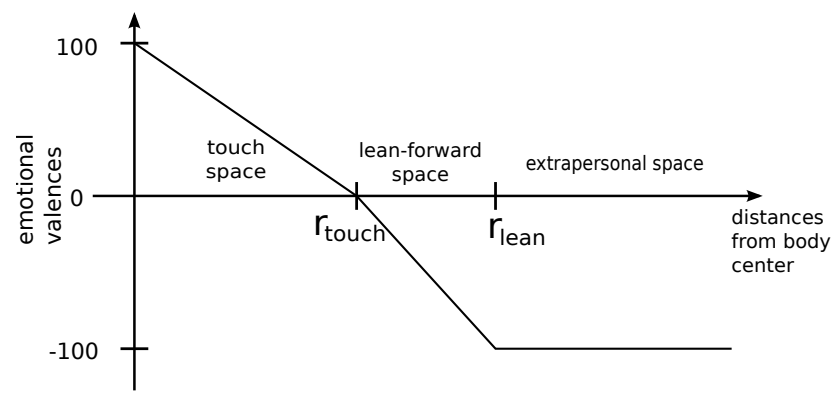

Fig. 2. The cost function maps spatial distances starting from the center of peripersonal space to emotional valences.

Max's game moves are performed by means of a sequence of plans defined in his BDI module. In order to place a block on the tower, Max first searches for the most appropriate one. Depending on the block's position, a corresponding emotional valence is triggered according to the cost function illustrated in Figure 2. This cost function associates peripersonal space distances and thus the physical effort of motor actions to emotional valences. The function reflects the effort of Max's reaching movements according to humans' physical effort. With increasing reach distance of objects, more physical effort is needed for humans to reach for them [17].

Blocks located in Max's touch space are easy to reach with less effort and lower cost, thus they are associated with emotional valences ranging in $[0,100]$ where the value of 100 corresponds to the center of this space. Blocks located in the lean-forward space are reachable but require more effort and higher cost, thus they are associated with emotional valences ranging in $[-100,0]$ where the value of 0 corresponds to the limiting border of touch space. Blocks located in extrapersonal space are not reachable, thus they are associated with emotional valences with a constant value of -100 . If the 
most appropriate block is not reachable for Max, he searches until he finds the next most appropriate and reachable one. Finally, Max places this block on the tower. This game move is defined as a goal success and is rewarded with a positive emotional valence. Otherwise, if no appropriate reachable block is found Max's turn is missed. This is defined as a goal failure and is rewarded with a negative emotional valence. In this scenario, cooperation consists of helping each other in accomplishing the game move. The helping action is defined as placing the most appropriate block toward a location reachable for the partner. Depending on the position of the placed block, i.e., how near the partner is placing the block to Max, an emotional valence is triggered according to the cost function shown in Figure 2.

\section{Empathic Behavior}

The work on the virtual human's empathic behavior is motivated by research in psychology and neuropsychology. Max's empathic behavior consists of three processing steps [5]: Empathy Mechanism, Empathy Modulation, and Expression of Empathy.

\subsection{Empathy Mechanism}

An empathic emotion can be elicited even if it is not felt or expressed by the partner (cf. [20]). Thus, in our spatial interaction scenario, the Empathy Mechanism by which an empathic emotion is produced is referred to as situational role-taking and is based on Max's spatial perspective-taking capabilities.

Spatial perspective-taking consists of Max modeling the partner's peripersonal space by projecting his own peripersonal space to the partner. Simulating the partner's perspective by using own body structure is known as embodied simulation [8] and is a hypothesis of how humans understand others. Thus, by means of his spatial perspectivetaking capabilities, Max infers the object positions in the partner's simulated peripersonal space.

Situational role-taking (cf. [4]) consists of generating a hypothesis about the partner's emotional state by appraising the partner's situation with the same appraisal mechanisms that Max would use if he were in this situation himself. In our spatial interaction task, during the partner's game turn Max infers the block positions in the partner's peripersonal space by means of his spatial perspective-taking capabilities and generates emotional valences for the partner in the same way as illustrated in Figure 2. These emotional valences drive the hypothesized partner's emotion dynamics which is simulated within Max's emotion simulation module. Thus, the hypothesized partner's emotional state consists of a PAD value and an intensity value of an activated primary emotion and is represented by an additional reference point in Max's emotion simulation module.

The elicitation of an empathic emotion is caused by detecting the occurrence of a desirable or a not desirable event for others [21]. Thus, an empathic emotion is elicited only if the partner's emotional state is positive/negative or rises/fails rapidly with respect to predefined thresholds. That is, with respect to a predefined short time interval $T$, the difference between inferred PAD values corresponding to the timestamps $t_{k-1}$ 
and $t_{k}$, with $t_{k}-t_{k-1}<=T$, is calculated as $\left|P A D_{t_{k}}-P A D_{t_{k-1}}\right|$. If this exceeds a predefined saliency threshold $T H$ or if $\left|P A D_{t_{k}}\right|$ exceeds a predefined saliency threshold $T H^{\prime}$, then the current emotional state $P A D_{t_{k}}$ and its related primary emotion represent the empathic emotion. Otherwise, no empathic emotion is elicited. The predefined thresholds can be interpreted as representing Max's responsiveness to the partner's situation.

\subsection{Empathy Modulation}

Based on [6] and [21], the empathic emotion produced by the Empathy Mechanism is modulated by the following factors: First, the empathizer's mood represented by Max's emotional state. Second, Max relationship to the partner as Max's liking toward his partner. Third, the situational context represented by deservingness as the degree to which the partner deserves/not deserves the event.

The modulation of the empathic emotion takes place in the PAD space and is realized by applying the following equation each time $t$ an empathic emotion is elicited:

$$
\begin{array}{r}
\text { empEmo }_{t, \text { mod }}=o w n E m o_{t}+ \\
\left(\text { empEmo }_{t}-\text { ownEmo }_{t}\right) *\left(\sum_{i=1}^{n} p_{i, t} * w_{i}\right) /\left(\sum_{i=1}^{n} w_{i}\right)
\end{array}
$$

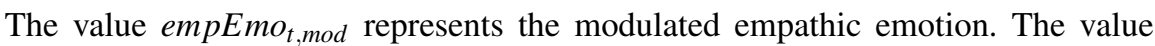

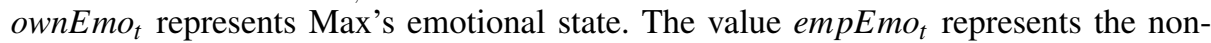
modulated empathic emotion resulting from the previous processing step. The values $p_{i, t}$ represent an arbitrary predefined number $n$ of modulation factors that could have values ranging in $[0,1]$ such as liking and deservingness. Thus in our scenario, $n=2$. Based on [21], liking and deservingness could be represented by values ranging in $[-1,1]$ from disliked, not-deserved to most-liked, most-deserved. The value 0 represents neither liked, deserved nor disliked, not-deserved. In this paper only positive values of liking and deservingness are considered. Note that positive values of deservingness represent deserved positive events and not deserved negative ones.

We define the degree of empathy as the distance between empEmo t,mod $_{\text {, }}$ and empEmo t $_{t}$

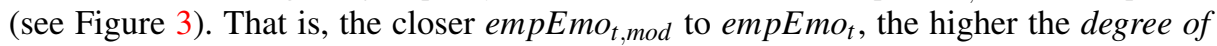

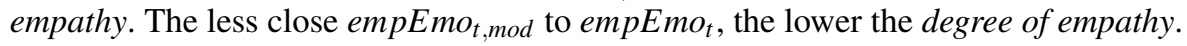

The impact of the modulation factors on the degree of empathy is as follows: The

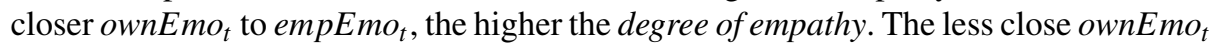

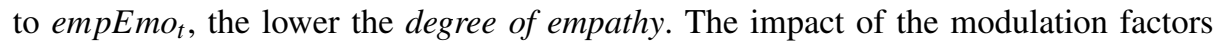
$p_{i, t}$ is calculated through a weighted mean of their current values at timestamp $t$. In our scenario, liking is defined as having more impact on the degree of empathy than deservingness and is thus weighted higher. The way how in our scenario the values of liking and deservingness are determined is introduced in Section 6. The higher the value of $p_{i, t}$ 's weighted mean, the higher the degree of empathy. The lower the value of $p_{i, t}$ 's weighted mean, the lower the degree of empathy.

Following [11], the empathic response to the other's emotion can be any emotional reaction compatible with the other's condition. Therefore, empEmo $o_{t, \text { mod }}$ is facilitated 


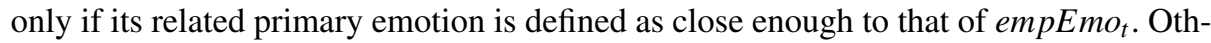
erwise, empEmo t,mod $_{\text {in }}$ inhibited. Primary emotions defined as close to empEmo ${ }_{t}$ 's primary emotion should represent emotional reactions that are compatible with the other's situation. 'Closeness' is not defined as Euclidean distance in PAD Space, but by defining thresholds for each of the dimensions individually. The choice of the thresholds is a matter of design and evaluation.

For example, Figure 3 shows Max's PA space of high dominance. At time $t_{k-1}$, ${ }_{\text {ownEmo }} \boldsymbol{t}_{t_{k-1}}$ has as related primary emotion happy, empEmo $t_{t_{k-1}}$ has as related primary emotion annoyed, and the weighted mean of $p_{i, t_{k-1}}$ is set to the value 0.4 . The resulting empEmo $_{t_{k-1}, \text { mod }}$ has as related primary emotion surprised which is defined as not close enough to annoyed. At this stage empEmo th-1 $_{t_{k-1}, \text { mod }}$ is inhibited and Max's expression of empathy is not triggered. At time $t_{k}$, ownEmo $_{t_{k}}$ is the neutral state concentrated, empEmo $t_{t_{k}}$ has as related primary emotion angry, and the weighted mean of $p_{i, t_{k}}$ is set to the value 0.6 . The resulting $e m p E m o_{t_{k}, \text { mod }}$ has as related primary emotion annoyed which is defined as close enough to angry. At this stage empEmo $t_{t_{k}, \text { mod }}$ is facilitated and the next processing step Expression of Empathy is triggered.

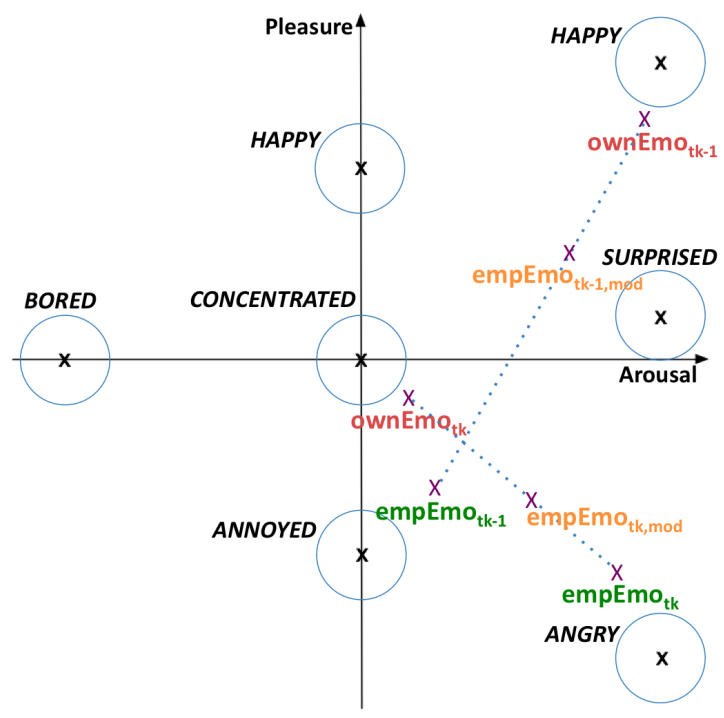

Fig. 3. Max's PA space of high dominance. The primary emotions happy, surprised, angry, annoyed, bored, and the neutral state concentrated are located at different PA values. The reference points ownEmo $_{t_{k-1}}$ and ownEmo $_{t_{k}}$ represent Max's emotional state at timestamps $t_{k-1}$ and $t_{k}$. The reference points empEmo $\boldsymbol{t}_{k-1}$ and $e m p E m o_{t_{k}}$ represent the non-modulated empathic emotion at timestamps $t_{k-1}$ and $t_{k}$. The reference points empEmo $t_{t_{k-1}, \text { mod }}$ and $e m p E m o_{t_{k}, \text { mod }}$ represent the modulated empathic emotion at timestamps $t_{k-1}$ and $t_{k}$. 


\subsection{Expression of Empathy}

Max's facial expressions are triggered by the intensity of the primary emotion of the modulated empathic emotion. In our spatial interaction scenario, Max's helping action is triggered only if the partner's pleasure becomes negative. Max can detect changes in the partner's pleasure values by calculating their difference, $P_{t_{k}}-P_{t_{k-1}}$, at timestamps $t_{k-1}$ and $t_{k}$. If $P_{t_{k}}-P_{t_{k-1}}<=0$ and $P_{t_{k}}<=0$, a helping action is triggered. Max's helping action is modulated by its degree of empathy. The calculation of Max's degree of empathy as well as its impact on Max's helping action are introduced in the next section.

\section{Modulating a Virtual Human's Cooperative Spatial Behavior}

The calculation of the modulation factor values liking, deservingness and degree of empathy are introduced in this section. Further, the impact of the degree of empathy on Max's helping action is introduced.

Liking The degree to which Max likes his interaction partner is calculated as the assumed partner's degree of empathy with Max. That is, the more the partner empathizes with Max, the more Max likes his partner. Based on the way Max's degree of empathy influences his helping actions (see Equation 4), he generates a hypothesis about the partner's degree of empathy from the partner's investment of helping actions.

Deservingness The degree to which the position of an appropriate block needed by the partner is a deserved or not deserved event is calculated as the number of reachable appropriate blocks in Max's touch space divided by the number of all existing appropriate blocks. That is, the more reachable appropriate blocks are in Max's touch space, the higher the value of deservingness and vice versa.

Degree of empathy The degree of empathy is defined as the distance between the modulated empathic emotion $e m p E m o_{t, m o d}$ and the non-modulated empathic emotion

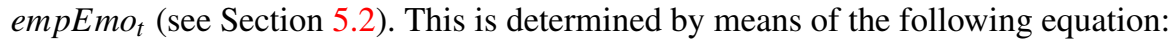

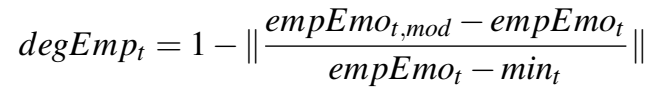

empEmo $_{t}$ denotes the maximum value an empEmo t,mod $_{\text {can }}$ have (see Figure 3). $\mathrm{min}_{t}$ denotes the minimum value from that empEmo t,mod $_{\text {is }}$ facilitated (see Section 5.2) and lies on the straight line spanned by ownEmo $_{t}$ and empEmo ${ }_{t}$. The degree of empathy $\operatorname{degEmp} p_{t}$ has values ranging in $[0,1]$.

Degree of helping Each time a helping action is triggered, Max places the most appropriate block to a position where the partner can reach it, i.e., to a position in interaction space illustrated as grey area in Figure 1, right. Interaction space's boundary is determined by two circular arcs spanned by the lean-forward spaces of Max and his partner. We denote the circular arc spanned by Max as $\operatorname{leanArc}_{\text {Max }}$ and that of his partner

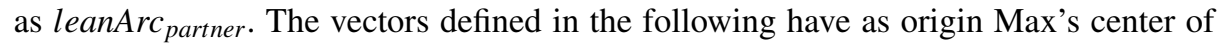
peripersonal space (see Section 3.2). The closest position $P_{m}$ to the partner where Max can place a block is defined as the intersection of $\operatorname{leanArc}_{\text {Max }}$ and the line segment spanned by Max's center of peripersonal space and the partner's center of peripersonal space. The position vector of $P_{m}$ is denoted by $\mathbf{p}_{m}$. Depending on Max's degree of 
empathy degEmp, his helping action is modulated. That is, degEmp determines how near the most appropriate block with vector $\mathbf{p}_{b}$ is placed toward the partner. With the following equation the new position $\mathbf{p}_{b N e w}$ of the block is calculated:

$$
\mathbf{p}_{b N e w}=\mathbf{p}_{b}+\left(\operatorname{degEmp} * \mathbf{p}_{\text {help }}\right) \text {, with } \mathbf{p}_{\text {help }}=\mathbf{p}_{m}-\mathbf{p}_{b}
$$

Equation 4 applies only if the block is located within interaction space, otherwise $\mathbf{p}_{b}$ is set to the intersection point of leanArc partner and the line segment spanned by $P_{m}$ and $\mathbf{p}_{b}$. This assures that $\operatorname{degEmp}$ only modulates the part of $\mathbf{p}_{\text {help }}$ in interaction space that contributes to a helping action. That means, our approach avoids helping actions by which blocks are placed outside interaction space, where the partner still cannot reach them and may judge Max to be non-cooperative. To control the actual placing motion two values of the potential field (see Equation ??) have to be specified: the radius $r_{\text {touch }}$ is set to the length of $\mathbf{p}_{b N e w}$ and the strength $\xi_{\text {peri }}$ is set to degEmp. Thus, the distance and velocity of the placing-motion are modulated. Therefore, the higher Max's degree of empathy, the faster and nearer to his partner, he places blocks in the partner's peripersonal space. For example, the maximum value of $\operatorname{degEmp}=1$ leads to a potential field where $r_{\text {touch }}$ is set to the maximum value of $r_{\text {lean }}$ and $\xi_{\text {peri }}$ is set to a predefined maximum velocity. In this case, Max performs the most helpful action by quickly bending the torso to lean forward and placing the block at $P_{m}$ closest to the partner. The example shows that Max would only perform a motor action associated with more cost, if his degree of empathy with the partner is high.

Summarized Task Course At the beginning of the tower building task Max's values of liking and deservingness are set to the minimum value of 0. Max's empathy for the partner increases with following factors: with increasing liking value, i.e the more the partner helps Max by placing needed blocks closer to Max; with increasing deservingness value, i.e the more reachable appropriate blocks Max has than the partner; and when the similarity between Max's and the partner's mood becomes higher. The more Max empathizes with the partner, the closer and faster he places needed blocks in front of the partner and thus the more effort he invests in helping him.

\section{Conclusion and Future Work}

In this paper we presented an approach for triggering and modulating the virtual human Max's helping actions in a cooperative spatial interaction task depending on his degree of empathy with his partner. Max's helping action enables his partner to put the most appropriate block on the tower. Thus, the height of the resulting tower implicitly reflects the amount of cooperation in the course of the task. This may be interpreted as follows: The more the partners share their emotions, the more they share their space to help each other. The more Max leans forward to place objects near his partner, the more Max expresses his empathy. This is in agreement with Jones and Wirtz [12], who state that human approach behaviors such as leaning forward, have been shown to convey positive affect, involvement and liking. While this paper focuses more on the technical details of the proposed approach, in future work an evaluation of this approach is planned to investigate how Max's modulated helping actions impact a human partner's engagement in achieving a successful cooperation. Since the more the partner helps Max, the more 
Max likes his partner, empathizes, and helps him, we expect that in order for the human partner to successfully solve the building task with Max, the human partner will show more engagement in cooperating with and helping Max.

Moreover, a challenging work in the future is to model Max's competitive behavior triggered by his negative empathy. This can be realized by modeling the peripersonal space field as an attraction field and by considering negative values for the liking and deservingness factors. This extension may reveal Max's modulated competitive behavior by placing all blocks from interaction space in his peripersonal space, where only he can access them.

It is conceivable to extend the current model within a more complex scenario of a cooperative city planning game developed in our research group. This scenario consists of a city grid map placed on a table between two interaction partners, thus forming a near-space interaction. The game consists of planning a city by placing buildings on the city grid map where the interaction partners can have joint or interfering goals. Their joint goals are to take the citizens' preferences into account while their interfering goals are to take their own preferences into account when planning the city. The interaction partners' and citizens' preferences consist of security, ecology, and amusement represented by different building types. Again, in this scenario, the virtual human's helping action is defined as relocating the most appropriate, in this case the most preferred object to positions reachable for the partner. The helping action is triggered and modulated by the virtual human's degree of empathy with its partner. A challenge will be to find the best position fitting the degree of empathy even when the determined position is occupied by another object or where the agent is not allowed to place an object during the partner's turn. Individual role-taking [10] will be used in order to find out which object is preferred by the interaction partner. This is defined as the ability to generate a hypothesis about the interaction partner's emotional state by taking, e.g, his preferences, goals, beliefs into account.

\section{Acknowledgments}

This research is kindly supported by the Deutsche Forschungsgemeinschaft (DFG) in the Collaborative Research Center 673. This paper is a preprint version of an article published by Springer-Verlag. The original publication is available at

http://link.springer.com/chapter/10.1007/978-3-642-23974-8_38.

\section{References}

1. R. Aylett and S. Louchart. If i were you: double appraisal in affective agents. In in Proceedings of 7th International Conference on Autonomous Agents and Multiagent Systems (AAMAS 2008), page 12331236, Estoril, Portugal.

2. C. Becker, N. Lessmann, S. Kopp, and I. Wachsmuth. Connecting feelings and thoughts modeling the interaction of emotion and cognition in embodied agents. In Seventh International Conference on Cognitive Modeling (ICCM-06), pages 32-37. Edizioni Goliardiche, 2006.

3. C. Becker-Asano and I. Wachsmuth. Affective computing with primary and secondary emotions in a virtual human. Autonomous Agents and Multi-Agent Systems, 2009. 
4. H. Boukricha, C. Becker, and I. Wachsmuth. Simulating empathy for the virtual human max. In 2nd International Workshop on Emotion and Computing, in conj. with the German Conference on Artificial Intelligence (KI2007), Osnabrück, Germany, pages 22-27, 2007.

5. H. Boukricha and I. Wachsmuth. Empathy-based emotional alignment for a virtual human: A three-step approach. German Journal on Artificial Intelligence, Künstl Intell, 2011.

6. F. de Vignemont and T. Singer. The empathic brain: how, when and why? Trends in Cognitive Sciences, 10(10):435-441, 2006.

7. M. E. Foster, M. Giuliani, A. Isard, C. Matheson, J. Oberlander, and A. Knoll. Evaluating description and reference strategies in a cooperative human-robot dialogue system. In Proceedings of the 21st international joint conference on Artifical intelligence, pages 18181823, San Francisco, CA, USA, 2009. Morgan Kaufmann Publishers Inc.

8. V. Gallese. Embodied simulation: From neurons to phenomenal experience. Phenomenology and the Cognitive Sciences, 4(1):23-48, 2005.

9. J. Gray, C. Breazeal, M. Berlin, A. Brooks, and J. Lieberman. Action parsing and goal inference using self as simulator. In Proceedings of Fourteenth IEEE Workshop on Robot and Human Interactive Communication (Ro-Man05), pages 202-209. IEEE, 2005.

10. E. T. Higgins. Role-taking and social judgment: Alternative developmental perspectives and processes. In J. H. Flavell and L. Ross, editors, Social cognitive development: Frontiers and possible futures, pages 119-153. Cambridge University Press, Cambridge, England, 1981.

11. M. L. Hoffman. Empathy and Moral Development. Cambridge University Press, 2000.

12. S. M. Jones and J. G. Wirtz. How does the comforting process work? an empirical test of an appraisal-based model of comforting. Human Communication Research, 32(3):217-243, 2006.

13. A. Kendon. Conducting Interaction. Cambridge University Press, London, 1990.

14. O. Khatib. Real-time obstacle avoidance for manipulators and mobile robots. Int. J. Rob. Res., 5(1):90-98, 1986.

15. S. Kopp, B. Jung, N. Lessmann, and I. Wachsmuth. Max - a multimodal assistant in virtual reality construction. KI Zeitschrift (German Journal of Artificial Intelligence), Special Issue on Embodied Conversational Agents, 4/03:11-17, 2003.

16. N. Lessmann, S. Kopp, and I. Wachsmuth. Situated interaction with a virtual human - perception, action, and cognition. In G. Rickheit and I. Wachsmuth, editors, Situated Communication, pages 287-323. Mouton de Gruyter, Berlin, 2006.

17. L. S. Mark, K. Nemeth, D. Gardner, M. J. Dainoff, J. Paasche, M. Duffy, and K. Grandt. Postural dynamics and the preferred critical boundary for visually guided reaching. Journal of Experimental Psychology: Human Perception and Performance, 23(5):1365-1379, 1997.

18. S. McQuiggan, J. Robison, R. Phillips, and J. Lester. Modeling parallel and reactive empathy in virtual agents: An inductive approach. In Padgham, Parkes, Müller, and Parson, editors, Proc. of 7th Int. Conf. on Autonomous Agents and Multiagent Systems (AAMAS 2008), pages 167-174, Estoril, Portugal, 2008.

19. N. Nguyen and I. Wachsmuth. From body space to interaction space - modeling spatial cooperation for virtual humans. In Tumer, Yolum, Sonenberg, and Stone, editors, Proc. of the 10th Int. Conf. on Autonomous Agents and Multiagent Systems, AAMAS 2011, pages 1047-1054, 2011.

20. M. Ochs, C. Pelachaud, and D. Sadek. An empathic virtual dialog agent to improve humanmachine interaction. In Padgham, Parkers, Müller, and Parson, editors, Proc. of 7th Int. Conf. on Autonomous Agents and Multiagent Systems (AAMAS 2008), pages 89-96, Estoril, Portugal, 2008.

21. A. Ortony, G. Clore, and A. Collins. The Cognitive Structure of Emotions. Cambridge University Press, 1988. 
22. C. Pedica and H. H. Vilhjálmsson. Spontaneous avatar behavior for human territoriality. In Proc. of the 9th Int. Conf. on Intelligent Virtual Agents, IVA '09, pages 344-357, Berlin, Heidelberg, 2009. Springer-Verlag.

23. H. Prendinger and M. Ishizuka. The empathic companion: A character-based interface that adresses users' affective states. Applied Artificial Intelligence, 19:267-285, 2005.

24. S. H. Rodrigues, S. Mascarenhas, J. Dias, and A. Paiva. I can feel it too! : Emergent empathic reactions between synthetic characters. In 3rd International Conference on Affective Computing and Intelligent Interaction and Workshops, ACII, Amsterdam, Netherland, 2009. IEEE.

25. E. A. Sisbot, L. F. Marin, R. Alami, and T. Simeon. A mobile robot that performs human acceptable motion. In Proc in (IEEE/RSJ) Int. Conf. on Intelligent Robots and Systems, 2006. 